\title{
A NOVEL REAL-TIME SELF-SIMILAR TRAFFIC DETECTOR/FILTER TO IMPROVE THE RELIABILITY OF A TCP BASED END-TO-END CLIENT/SERVER INTERACTION PATH FOR SHORTER ROUNDTRIP TIME
}

\author{
Wilfred W. K. Lin, Allan K. Y. Wong, Richard S.L. Wu \\ Department of Computing, The Hong Kong Polytechnic University, Hung Hom, Kowloon, Hong Kong S.A.R. \\ Email:cswklin@comp.polyu.edu.hk,csalwong@comp.polyu.edu.hk,csslwu@comp.polyu.edu.hk \\ Tharam S. Dillon \\ Faculty of Infornation Technology, University of Technology Sydney, Broadway, Sydney, Australia \\ Email: tharam@it.uts.edu.au
}

\begin{abstract}
Keywords: real-time traffic pattern detection (RTPD), stationary, asymptotically second-order self-similarity, CAB, Gaussian property, fractal

Abstract: $\quad$ The self-similarity $\left(S^{2}\right)$ filter is proposed for real-time applications. It can be used independently or as an extra component for the enhanced RTPD (real-time traffic pattern detector) or E-RTPD. The $S^{2}$ filter basis is the "asymptotically second-order self-similarity" concept (alternatively called statistical $2^{\text {nd }} O S S$ or $S 2^{\text {nd }} O S S$ ) for stationary time series. The focus is the IAT (inter-arrival times) traffic. The filter is original because similar approaches are not found in the literature for detecting selfsimilar traffic patterns on the fly. Different experiments confirm that with help form the $S^{2}$ filter the FLC (Fuzzy Logic Controller) dynamic buffer size tuner control more accurately. As a result the FLC improves the reliability of the client/server interaction path leading to shorter roundtrip time (RTT).
\end{abstract}

\section{INTRODUCTION}

It is hard to harness the roundtrip time (RTT) of an end-to-end client/server interaction path over a TCP channel in time-critical applications. The problem is the heterogeneity and sheer size of the Internet. If the path error probability for retransmissions is $\rho$, the average number of trials (ANT) for successful transmission is

$$
\sum_{j=1}^{\infty} j\left[\rho^{j-1}(1-\rho)\right] \approx \lim _{j \rightarrow \infty} 1 /(1-\rho)
$$

The value $\rho$ encapsulates different faults and errors, and one of them is caused by buffer overflow along the end-to-end interaction path. There are two levels of buffer overflows: a) system/router level that includes all activities inside the TCP channel, and b) user level that involves the buffer at the receiving end. Methods to prevent network congestion that causes router buffer overflow include active queue management (AQM) (Braden, 1998). One effective approach to eliminate user- level buffer overflow to improve the end-to-end path reliability is dynamic buffer size tuning (Wong, 2002). The accuracy and stability of the tuning process, however, are affected by the Internet traffic patterns in terms of messages' inter-arrival times (IAT). To resolve this problem the previous realtime traffic pattern detector (RTPD) (Lin, 2004) was proposed. With the detected results the dynamic buffer size tuners can mitigate/nullify the ill effects by traffic on system stability and performance in a dynamic fashion. The RTPD, however, does not detect self-similar traffic, and this leads to the proposal of the self-similarity $\left(S^{2}\right)$ filter in this paper. Inclusion of the $S^{2}$ filter into RTPD created the enhanced RTPD (E-RTPD). It will be demonstrated later how E-RTPD helps the Fuzzy Logic Controller (Lin, 2004B) self-tune better on the fly to gain more accurate and smoother user-level dynamic buffer size tuning and shorter RTT as a result.

The Internet involves many different client/server interaction protocols (Lewandowski, 1998), and its 
traffic follows the power law (Medina, 2000). Over time the traffic in any part of the Internet may change suddenly, for example, from LRD (longrange dependence) to SRD (short-range dependence) or vice versa (Willinger, 2003). Using the Hurst $(\mathrm{H})$ effect (i.e. $H_{s s}$ (Taqqu, 2003)) as the yardstick then $0.5<H<1$ is for LRD and $0<H \leq 0.5$ for SRD. If $X^{m}=\left\{X_{l}^{m}: l \geq 1\right\}$ is a time series aggregate of size $m$ in a stochastic process $\mathrm{X}$, its autocorrelation function (ACF) (correleogram) is $r^{*}(1)=\sum_{i=1}^{N} r^{m}$, where $r^{m}$ is the autocorrelation of $X^{m}$ and $l$ for the aggregate level. The ACF of LRD traffic is non-summable (i.e. $\sum_{l-1}^{N \rightarrow \infty} r^{m} \approx \infty$ ), but it is summable for SRD (i.e. $\left.\sum_{i=1}^{N \rightarrow \infty} r^{m}<\infty\right)$.

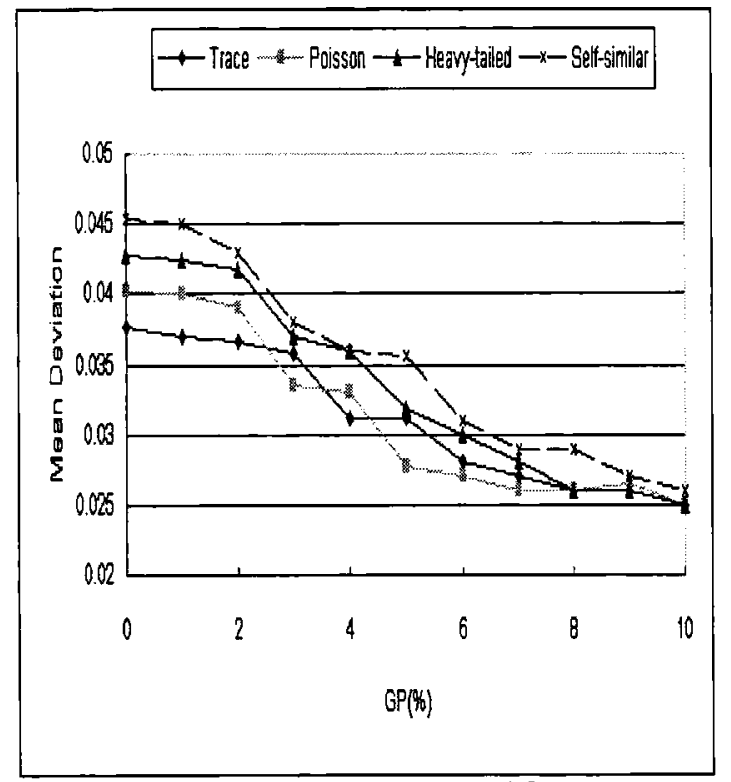

Figure 1: Internet traffic impact on FLC accuracy

It is impractical to monitor the overwhelming number of network parameters in the Internet to harness the client/server RTT. A practical approach is to treat the Internet as a "black box" and measure the end-to-end RTT to interpret the channel behavior. This is the IEPM (Internet End-to-End Performance Measurement (Cottrel, 1999)) approach. Any sudden changes in the IAT traffic pattern affect the performance of applications running on the Internet. The traffic's ill effect on the FLC stability and accuracy (Lin, 2004) is an example. Figure 1 shows how the mean deviations
(MD) from the FLC steady-state reference due to traffic changes in one deployment. Traffic selfsimilarity (or self-affinity) consistently produces the largest deviations compared to heavy-tailed and Markovian traffic. Two objects are geometrically similar if one is derived from another by linear scaling, rotation or translation. The GP\% (gradient percentage) in Figure 1 is a derivative (D) control parameter in FLC. For the same GP value different traffic patterns produce different $M D$ values. The reconfigurable version of the FLC uses the RTPD to detect a traffic pattern on the fly and utilizes the result to neutralize traffic ill effects by choosing the correct GP value accordingly (Lin, 2004). The RTPD differentiates LRD from SRD and identifies heavy-tailed traffic, but it does not detect selfsimilar patterns. Combining the previous RTPD model with the self-similarity $\left(S^{2}\right)$ filter (or simply $S^{2}$ filter) creates the enhanced RTPD (E-RTPD), which has the capability to identify self-similarity and compute its dimension (D). If an object is geometrically, recursively split into similar pieces, then at the $K^{\text {th }}$ iteration step the total measure of the object is "product of the number of similar pieces and $O^{D}$ ". The parameter $O$ is the splitting resolution or reduction. The Cantor $\mathrm{Set}$ is an example in which a line segment of interval $[0,1]$ is drawn as the first step (i.e. $K=0$ ). This line is then manipulated by the subsequent steps: a) divide the line into three equal portions (i.e. resolution is $1 / 3$ ) and remove the middle portion (i.e. $K=1$ ), b) remove the middle portions from the remaining two (i.e. $K=2$ ), and c) repeat the last step ad infinitum. The $K^{\text {th }}$ iteration produces $2^{K}$ similar line segments of length $s=(1 / 3)^{K}$ each. The Cantor Set's selfsimilarity dimension is defined by the formula $D_{s}=2^{K} *(1 / 3)^{K}$ or alternatively

$$
D_{s}=[(K \log (2)) /(K \log (3))] \approx 0.63
$$

An object is fractal if its $D$ value is non-integer. Different non-converging dimension definitions exist, the Cantor Set provides only a conceptual basis. A stochastic process $X(t)$ is $H_{s s}$, selfsimilar and fractal, provided that its two finitedimensional distributions, $X(a t)$ and $a^{H} X(t)$ are identical for $a>0$. That is, the following expression holds: 
$\left\{X\left(a t_{1}\right), X\left(a t_{2}\right) \ldots X\left(a t_{n}\right)\right\} \equiv\left\{a^{H} X\left(t_{1}\right), \ldots a^{H} X\left(a t_{n}\right)\right\} ;$ $\equiv$ means equality and $\mathrm{H}$ is the scaling exponent.

\section{RELATED WORK}

The previous RTPD is enhanced from the traditional $\mathrm{R} / \mathrm{S}$ (rescaled adjusted statistics) approach for nonreal-time (i.e. "post-mortem") applications. The enhanced R/S (i.e. E-R/S) is a real-time “ $M^{3} R T+R / S+$ filtration " package.

The $M^{3} R T$ element is a micro Convergence Algorithm (CA) or MCA implementation (Wong, 2001). The $C A$ is the technique adopted from the IEPM (Internet End-to-End Performance Measurement) problem domain (Cottrel, 1999). The MCA, which predicts the mean of a traffic waveform quickly and accurately, operates as a logical object to be invoked for service anytime and anywhere by message passing. It helps the R/S mechanism differentiates SRD from LRD on-line. The filtration process activates an appropriate filter to identify the exact traffic pattern; for example, the modified QQ-plot filter identifies heavy-tailed distributions. The main RTPD contribution is that it can be a part of any time-critical application, which uses it to detect traffic patterns on the fly. These applications can then use the detected result to selftune for better system performance (Lin, 2004). Similar to its R/S predecessor, the E-R/S calculates the Hurst $(\mathrm{H})$ parameter/value but on-line. The $0.5<H<1$ range means LRD traffic (e.g. heavytailed and self-similar traces) and $0<H \leq 0.5$ for SRD (short-range dependence, e.g. Markovian traffic) (Molnar, 1999).

The traditional R/S is defined by

$R / S=\frac{\max \left\{W_{i}: i=1,2, \ldots, k\right\}-\min \left\{W_{i}: i=1,2, \ldots, k\right\}}{\sqrt{\operatorname{var}(X)}}$

The parameter $W_{i}$ is computed as

$W_{i}=\sum_{m=1}^{i}\left(X_{m}-\bar{X}\right)$

for $\mathrm{i}=1,2, \ldots \mathrm{k}$, where $\bar{X}$ is the mean of. $\bar{X}=1 / k \sum_{i=1}^{k} X_{i}$

The best value for $k$ is usually found by trial and error, and this becomes the drawback because R/S accuracy and speed depend on $k$. The $\mathrm{R} / \mathrm{S}$ ratio is the rescaled range of the stochastic process $\mathrm{X}$ over a time interval $k,\left\{X_{i}: i=1,2, \ldots k\right\}$. A useful $\mathrm{R} / \mathrm{S}$ feature is the $\log -\log$ of $R / S \approx(k / 2)^{H}$, which yields the $H$ value.

The CA operation, which is derived from the Central Limit Theorem, is summarized by the equations: (2.1) and (2.2). The estimated mean $M_{i}$ in the $i^{t h}$ prediction cycle is based on the fixed $F$ (flush limit) number of data samples. The cycle time therefore depends on the interval for collecting the $F$ samples. It was confirmed previously that $M_{i}$ has the fastest convergence for $\mathrm{F}=14$ (Wong, 2001). Other parameters include: a) $M_{i-1}$ is the feedback of the last predicted mean to the current $M_{i}$ prediction cycle, b) $m_{j}^{i}$ is the $j^{\text {th }}$ data item sampled in the current $i^{\text {th }} M_{i}$ cycle, $j=1,2,3, \ldots,(F-1)$, and c) $M_{0}$ is the first data sample when the MCA had started running. In the E-R/S, $M_{i}$ replaces $\bar{X}$ to yield.

$W_{i}=\sum_{m=1}^{i}\left(X_{m}-M_{i}\right)$

This replacement makes the E-R/S more suitable for real-time applications because the number of data items (e.g. IAT) to calculate $W_{i}$ becomes predictable (i.e. $F=14$ ). In real-life applications $\vec{X}=1 / k \sum_{i=1}^{k} X_{i}$ will need much longer computation time than $M_{i}$ for two reasons: a) $k$ is usually larger than $F$, and b) the IAT among $X_{i}$ could be so large that the product of " $k$ and average IAT" means a significant time delay. In an E-RTPD implementation the E-/RS, $M^{3} R T$ and filter modules are running in parallel. The E-RTPD execution time depends on the E-R/S module, which has the longest execution. The Intel's VTune Performance Analyzer (Intel VTune, 2002) records from the Java RTPD prototype the following average execution times in clock cycles: 981 for E$\mathrm{R} / \mathrm{S}, 250$ for $M^{3} R T$, and 520 for the modified $Q Q$ plot filter. The novel $S^{2}$ filter provides RTPD with the additional capability to quickly detect selfsimilar traffic on the fly.

$$
\begin{aligned}
& M_{i}=\frac{M_{i-1}+\sum_{j=1}^{j=F-1} m_{j}^{i}}{F} \ldots \ldots \ldots(2.1) ; \\
& M_{0}=m_{j=0}^{i=1} \ldots \ldots \ldots .(2.2) ; i \geq 1
\end{aligned}
$$




\section{THE SELF-SIMILARITY FILTER}

LRD traffic has at two basic components: heavytailed and self-similar. The proposed self-similarity $\left(S^{2}\right)$ filter differentiates heavy-tailed IAT patterns from self-similar ones. Self-similarity in many fractal point processes results from heavy-tailed distributions, for example, FRP (Fractal Renewal Process) inter-arrival times. The heavy-tailed property, however, is not a necessary condition for self-similarity because at least the FSNDPP (Fractal-Shot-Noise-Driven Poisson Process) does not have heavy-tailed property. The $S^{2}$ filter basis is the "asymptotically second-order self-similarity" concept, or simply called statistical $2^{\text {nd }} O S S$ or $S 2^{\text {nd }} O S S$, which associates with a sufficiently large aggregate level or lag $l$ in a stochastic process $\mathrm{X}$. For an aggregate $X^{m}=\left\{X_{l}^{m}: l \geq 1\right\}$ of size $m$ in $\mathrm{X}, S 2^{\text {nd }} O S S$ for $m \rightarrow \infty$ means that the associated autocorrelation function (ACF), namely $r^{m}(l)$ (for $X^{m}$ ) is proportional to $l^{-(2-2 H)} . S 2^{n d} O S S$ is LRD for its ACF is nonsummable, as indicated by .

$$
r^{m}(l)=\sum_{l-1}^{\infty} r^{m}=\infty
$$

The condition of " $r^{m}(l) \propto l^{-(2-2 H)}$ for $m \rightarrow \infty$ " is mathematically equivalent to the slowly decaying variance property. That is, the variance of the mean of sample size $m$ decays more slowly than $m$. This phenomenon is represented by the expression: $\operatorname{Var}\left(X^{m}\right) \propto m^{-\beta}$. For a stationary $2^{\text {nd }} O S S$ process $\mathrm{X}$ and $0.5<H<1$ the value of $\beta=2-2 H$ should apply. Equations (3.1) and

(3.2) summarize the $S 2^{\text {nd }} O S S$ property and they hold for the weaker condition in equation (3.3). The slowly decaying variance property is clear if a loglog plot is produced for equation (3.1). As shown by equation (3.4), $\log (\operatorname{Var}(X))$ is a constant, $\log \left(\operatorname{Var}\left(X^{m}\right)\right)$ versus $\log (m)$ yields a straight line with slope $-\beta$. The $H$ value can then be calculated by the

$H=1-(\beta / 2)$ formula. The $S^{2}$ filter finds $\beta$ for $X^{m}$ on the fly. The $\operatorname{Var}\left(X^{m}\right)$ calculation uses the mean value $E\left(X^{m}\right)$ estimated by the $M^{3} R T$ process. $E\left(X^{m}\right)$ is $m^{-1} \sum_{n=(l-1) m+l}^{l m} X_{n}$ conceptually, and the key for the $S^{2}$ filter operation is to choose a sufficiently large $m$, which is the multiples (i.e. C) of $F=14$ to virtually satisfy $m \rightarrow \infty$; $m=C^{*} F$ for estimating $\beta$. The detected result is available at the $A g$ time point. In Figure 2 for example, the $\beta$ result for aggregate 2 is available at the point of $A g=2$.

$$
\begin{aligned}
& \operatorname{Var}\left(X^{m}\right)=\frac{1}{m^{(2-2 H)}} \operatorname{Var}(X) \ldots .(3.1) \\
& r^{m}(l)=r(k) \ldots .(3.2) \\
& \lim _{m \rightarrow \infty} r^{m}(l)=r(k) \ldots .(3.3) \\
& \log \left(\operatorname{Var}\left(X^{m}\right)\right)=\log (\operatorname{Var}(X))-\beta \log (m) \ldots(3.4)
\end{aligned}
$$

The process in the $S^{2}$ filter to calculate $\beta$ is the "continuous aggregate based (CAB)" method. The $\mathrm{CAB}$ evaluates if an aggregate is stationary by checking its Gaussian property or "Gaussianity" (Arvotham, 2001) by the kurtosis and skewness metrics. A symmetrical normal distribution has perfect Gaussianity indicated by kurtosis $=3$ and skewness $=0$. Statistically measured kurtosis and skewness values are rarely perfect, and reasonable limits can be used to indicate the presence of a bell curve, which belongs to the exponential family of independent stationary increments. The $S^{2}$ filter follows the $\mathrm{CAB}$ procedure and finds $\beta$ by linear regression, and the quality of which can be judged by the coefficient of determination or $R^{2}$ between 0 and 1 (Jain, 1992). Higher $R^{2}$ implies better quality for the linear regression. By the predefined threshold $T h_{R^{2}}$ (e.g. 0.85 or $85 \%$ ) the $S^{2}$ filter can reject a hypothesis of self-similarity in $X^{m}$ for $R^{2}<T h_{R^{2}}$. The CAB operation in Figure 2 works with the aggregates $X_{A g=1}^{m}$ in a stochastic process $\mathrm{X}$ along the time axis. Assuming: a) P1, P2, and P3 are the log-log plots for three successive aggregates based on equation (3.4), 
b) these plots yield different $\beta$ values: $\beta_{1}$ for $\mathrm{Pl}$ with $R^{2}=0.82, \beta_{2}$ for $\mathrm{P} 2$ with $R^{2}=0.98$, and $\beta_{3}$ for P3 with $R^{2}=0.95$, c) $A g=l$ is the aggregate level, and d) $T h_{R^{2}}=0.9$, then both $\mathrm{P} 2$ and P3 confirms self-similar traffic but not P1 (for $R^{2}<T h_{R^{2}}$ ). If $\mathrm{P} 2$ and $\mathrm{P} 3$ yield very different $\beta$ values, their $H$ values by

$H=1-(\beta / 2)$

indicate different dimensions or $\mathrm{D}$. The $\mathrm{D}$ value may change over time due to various factors, for example, the ON/OFF situations in the network (Willinger, 2003). A changing $\mathrm{D}$ or $\mathrm{H}$ is a sign of non-linearity in the stochastic process being examined. A D/H correlation will be demonstrated, but the focal discussion of how $\mathrm{H}$ or $\mathrm{D}$ could affect system stability will be left out.

Skewness is represented by $\frac{\sum_{i=1}^{N}\left(x_{i-\bar{x}}\right)^{3}}{(m-1) s d^{3}}$

, where $\bar{x}$ and $s d$ are the measured mean and standard deviation respectively for the aggregate of $m$ samples. It measures the symmetry of a bellshape aggregate distribution. A positive value indicates that the bell curve skews right and the right tail is heavier than the left one. Kurtosis is represented by

$$
\frac{\sum_{i=1}^{N}\left(x_{i}-\bar{x}\right)^{4}}{(m-1) s d^{4}}
$$

, and its value decides whether the bell curve is peaked (for positive value) or flat (or negative value) compared to the normal distribution with kurtosis $=3$ and skewness $=0$.

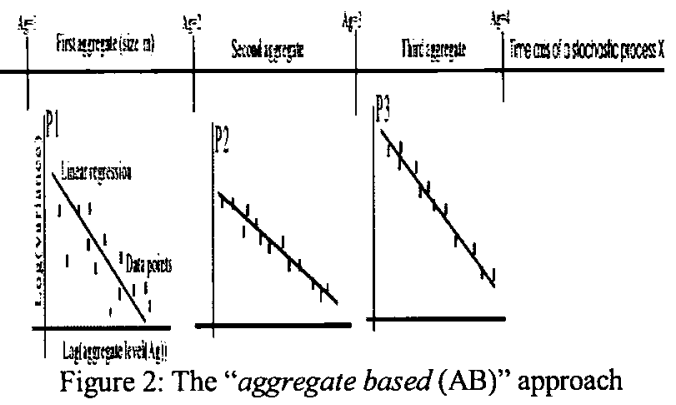

\section{EXPERIMENTAL RESULTS}

The $S^{2}$ filter was verified by simulations based on the CAB approach. The experiments were conducted on the stable Aglets mobile agent platform, which is designed for Internet applications. The Aglets makes the experimental results scalable for the open Internet. The setup for the experiments is shown in Figure 3, in which the driver and server are both aglets (agile applets). The driver picks a known waveform or a pre-collected IAT trace that may embeds different traffic patterns over time. The pick simulates the IAT among the requests that enter the server queue. The FLC dynamic buffer size tuner is the test-bed for the $S^{2}$ filter. It adjusts the buffer size on the fly by leveraging the current queue length, buffer length, and detected traffic pattern. The traffic pattern(s) that drives the IAT is also recorded by the E-RTPD that has included the $S^{2}$ filter. This helps matching the FLC control behavior with the specific traffic pattern. The VTune measures the E-RTPD's average execution time so that its contribution to time-critical applications on the Internet can be evaluated. Experiments with different IAT traffic patterns were carried out. The results conclude that the $S^{2}$ filter indeed detects self-similar traffic and helps the FLC deliver more accurate dynamic buffer size tuning. The experimental results presented here include: selfsimilarity detections, traffic and FLC accuracy, and D/H correlation.

Table 1 summarizes seven of the many different simulations conducted. The self-similar traces, which simulate the inter-arrival times (IAT) for the request into the server's buffer being controlled by the FLC (Figure 3), are generated by using the Kramer's tool (Kramer, 2002). The useful information from the Table 1 summary is listed as follows:

The $S^{2}$ filter always detect and recognizes selfsimilarity in the IAT traffic as long as the network loading or utilization $\psi$ is $50 \%$ (i.e. 0.5 simulated by the same tool) or less.

$\psi$ is proportional to the self-similarity dimension (explained later with Figure 9). For $\psi>0.4$ the traffic self-similarity scales differently as indicated Figure 5 and 6. Our analysis indicates that this is possibly the beginning of non-linear scaling or a sign of possible multifractal traffic. Both Figure 5 and 6 work with $T h_{R^{2}}=0.9$.

The scaling exponent $\mathrm{H}$ (Hurst effect) changes with $\psi$, which is inversely proportional to the IAT length that is the "reduction/resolution" in light of traffic. For $\psi \leq 0.4$ the scaling is basically the same (i.e. a monofractal sign). The $\beta$ value in 
A NOVEL REAL-TIME SELF-SIMILAR TRAFFIC DETECTOR/FILTER TO IMPROVE THE RELIABILITY OF A TCP BASED END-TO-END CLIENT/SERVER INTERACTION PATH FOR SHORTER ROUNDTRIP TIME

every case (row) in Table 1 is the average of several aggregates for the same stochastic process $\mathrm{X}$.

The kurtosis and skewness are different for the different self-similar traces. Nevertheless they always indicate the presence of a bell curve.

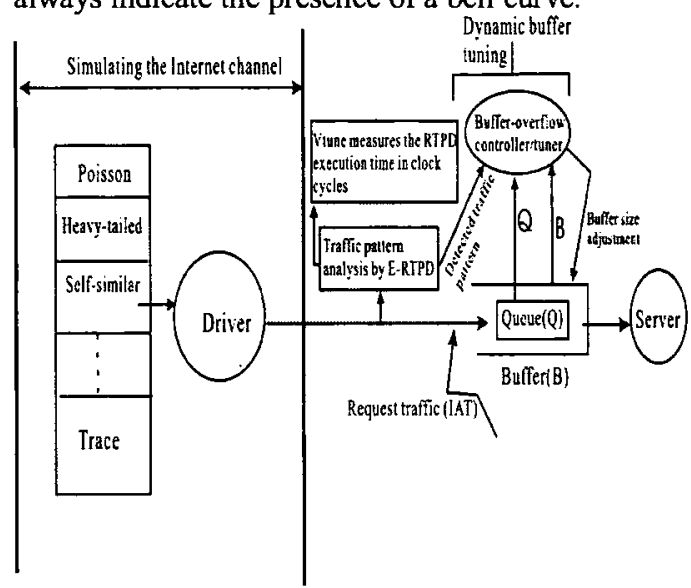

Figure 3: Setup for the $S^{2}$ filter experiments

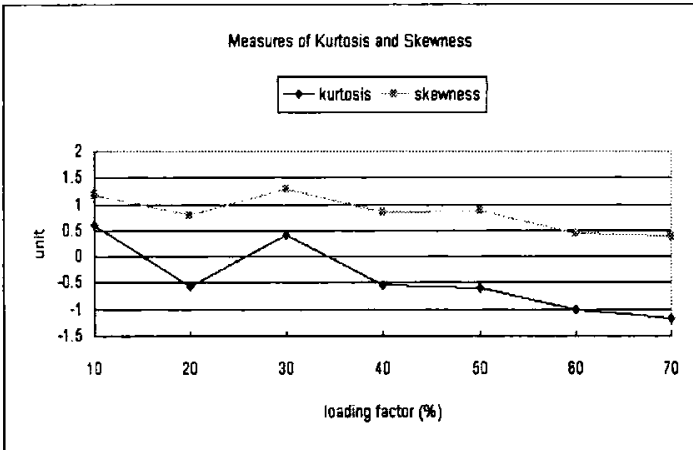

Figure 4: Kurtosis and skewness measurements

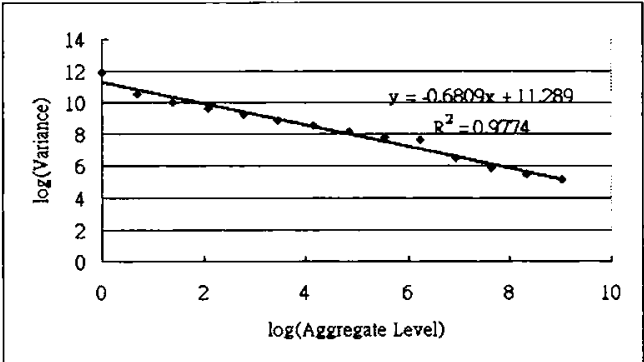

Figure 5: $S^{2}$ filter yields slope $=-0.6809(\beta=0.6809)$, $\mathrm{R}^{2}=97.74 \%$ for $\psi=0.2$

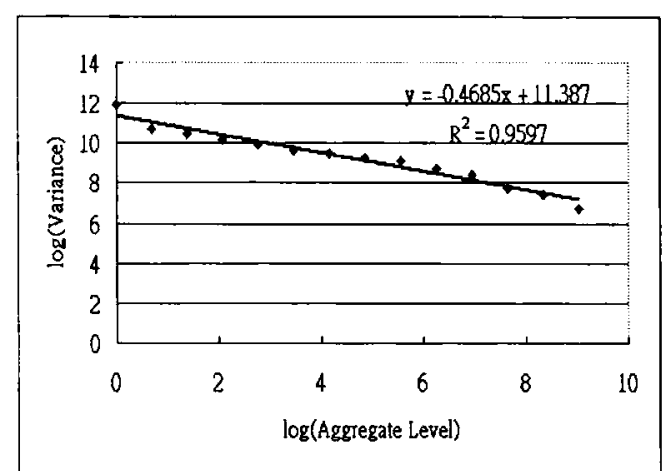

Figure 6: $S^{2}$ filter yields slope $=-0.4685(\beta=0.4685)$, $\mathrm{R}^{2}=95.97 \%$ for $\psi=0.5$

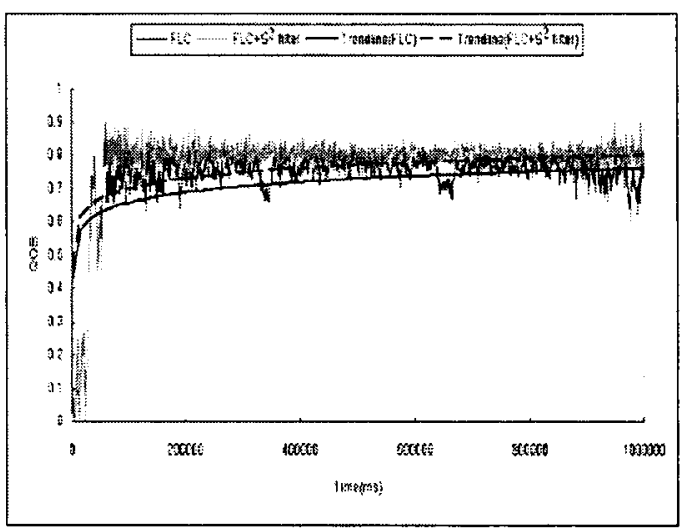

Figure 7: Faster convergence of the FLC $+S^{2}$ filter than the FLC working alone

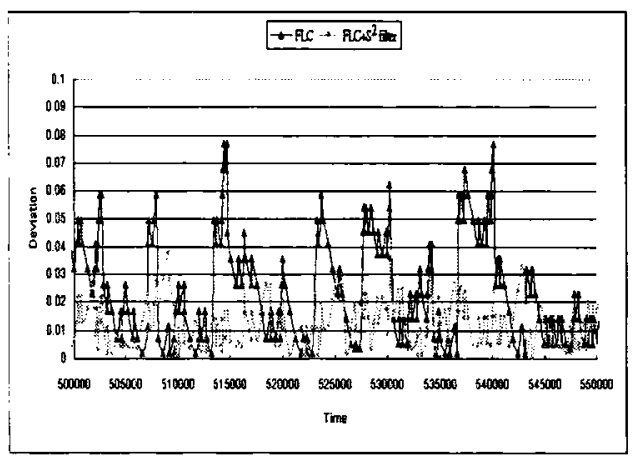

Figure 8: Less MD deviation by FLC+ $S^{2}$ than the FLC alone 


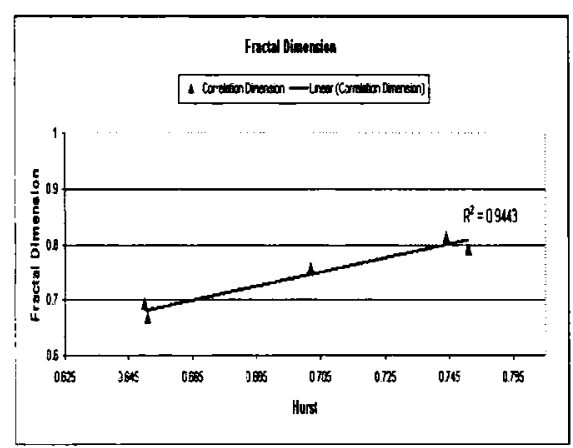

Figure 9: D/H correlation for Table 1

The kurtosis and skewness values for each case (row) in Table 1 are plotted for comparison (Figure 4). These values are obviously affected by the loading. When the loading is high (e.g. $60 \%$ and $70 \%$ ) the bell curve tends to skew less but still to the right. Meanwhile the bell curve tends to get flatter. Comparatively the skewness of the bell curves for the seven simulation cases in Table 1 are less than a Weibull ( gamma $=1.5$ ) distribution, which is relatively more peaked (kurtosis $\approx 4.5$ ). The trendlines in Figure 7 for the IAT traffic trace in Figure 5 shows that the "FLC $+S^{2}$ filter" combination converges much faster to given steady state than the FLC working alone. With help from the $S^{2}$ filter the FLC adjusts the GP value for the derivative (D) control element on the fly according to the currently detected self-similarity. As a result it produces less $\mathrm{MD}$ than the FLC working alone (Figure 8). In the experiments the FD3 tool (Sarraille, 2004), which confirms if an image (e.g. a time series generated by the Kramer's tool) is really fractal and measures its dimension $\mathrm{D}$, was used. The purpose is to evaluate the D/H correlations (Peitgen, 2004). This correlation for Table 1 is plotted and shown in Figure 9. It shows that if $\mathrm{D}$ changes suddenly, $\mathrm{H}$ also rescales accordingly to indicate possible traffic nonlinearity. In contrast, if $\mathrm{H}$ scales linearly, it is a sign of monfractal traffic. The intrinsic average $S^{2}$ filter execution time as observed from all the experiments is 1455 clock cycles as measured by the Intel's VTune Performance Analyzer. It is intrinsic because it works with immediately available data (without any actual IAT delay) in a trace. For a platform of 100 mega hertz the corresponding physical time is $1455 /\left(100 * 10^{6}\right)$ or 14.55 micro seconds. In real-life applications the $S^{2}$ filter has to collect enough IAT samples on the fly before computing $\beta$. This sampling latency can be significant, and therefore the success of $S^{2}$ filter application depends of choosing size $m$ for the $X^{m}$ aggregate correctly. For example, if the average IAT is one second, $m=1000$ means 1000 seconds. On the contrary for the same size $m$ and mean IAT of 1 $\mathrm{ms}$, the physical time is only one second. Therefore, the $m$ value for the $S^{2}$ filter Java prototype is a variable rather than a chosen constant, and the user/tester should fix the time span $T$ instead of collecting the fixed $m$ samples on the fly. That is, the number of samples (i.e. $m$ ) in an aggregate within $T$ depends on the IAT; shorter IAT delays yield a larger $m$. Then, the $S^{2}$ filter works adaptively with the $m$ value decided by the IAT for the "timed aggregate" based on the chosen $T$.

\section{CONCLUSION}

The novel self-similarity $\left(S^{2}\right)$ filter is proposed for real-time applications. It is based on the "asymptotically second-order self-similarity" concept (alternatively called statistical $2^{\text {nd }}$ OSS or $S 2^{\text {nd }} O S S$ ) for stationary time series. As a component in the enhanced RTPD or E-RTPD it helps the FLC dynamic buffer tuner yield more accurate control by detecting self-similarity in the IAT traffic. This means improved reliability for the client/server interaction path and shorter roundtrip time. The $S^{2}$ filter is original because there is no

Table 1: $S^{2}$ filter' $(\log$ (variance) versus $\log$ (aggregate level) to find $\beta$

\begin{tabular}{|l|c|l|l|l|l|}
\hline$\beta$ & $H=(1-\beta / 2)$ & $R^{2}$ (coefficient of determination) & loading $\psi$ & kurtosis & skewness \\
\hline 0.6583 & 0.671 & $0.956(95.6 \%)$ & $0.1(10 \%)$ & 0.597045 & 1.180861 \\
\hline 0.6809 & 0.660 & $0.975(97.5 \%)$ & 0.2 & -0.56218 & 0.798282 \\
\hline 0.6425 & 0.679 & $0.977(97.7 \%)$ & 0.3 & 0.40215 & 1.277175 \\
\hline 0.6473 & 0.677 & $0.972(97.2 \%)$ & 0.4 & -0.53386 & 0.861215 \\
\hline 0.4685 & 0.766 & $0.959(95.9 \%)$ & 0.5 & -0.58417 & 0.892037 \\
\hline 0.3762 & 0.812 & $0.885(88.5 \%)\left(\right.$ less than $\left.T_{R^{2}}\right)$ & $0.6($ rejected) & -1.01033 & 0.446756 \\
\hline
\end{tabular}


previous examples in the literature that can detect self-similarity in a time series on the fly. The next step in the research is to perfect the $\mathrm{CAB}$ approach by enabling it to determine the range of aggregate size $m$ that can produce accurate traffic detection but without any unnecessary and significant latency in the process.

\section{ACKNOWLEGEMENT}

The authors thank the Hong Kong PolyU and the Department of Computing for funding the RTPD research with grants APG51 and HJZ91.

\section{REFERENCES}

S. Arvotham, R. Riedi and R. Barabniuk, ConnectionLevel Analysis and Modeling of Network Traffic, Proc. of the IEEE/ACM Internet Measurement Workshop, 2001

B. Braden et al., Recommendation on Queue Management and Congestion Avoidance in the Internet, RFC2309, April 1998

L. Cottrel, M. Zekauskas, H. Uijterwaal, and T. McGregor, Comparison of Some Internet Active Endto-End Performance Measurement Projects, http://www.slac.stanford.edu/comp/net/wanmon/iepm-cf.html, 1999 Intel's VTune Performance Analyzer,

http://ww.intel.com/support/performancetools/vtune/v 5

R. Jain, The Art of Computer Systems Performance Analysis - Techniques for Experimental Design, Measurement, Simulation, and Modeling, Wiley, 1992

Kramer, Generator of Self-Similar Network Traffic, http://wwwcsif.cs.ucdavis.edu/ kramer/code/trf_gen I. html

S.M. Lewandowski, Frameworks for Component-based Client/Server Computing, ACM Computing Surveys, 30(1), March 1998, 3-27

Wilfred W. K. Lin, Richard S.L. Wu Allan K. Y. Wong, and Tharam S. Dillon, A Novel Real-Time Traffic Pattern Detector for Internet Applications, Proc. of the Australasian Telecommunication Networks and Applications Conference, Sydney, Australia (ATNAC'04), Dec 2004, 224-227

Wilfred W. K. Lin, Allan K. Y. Wong, and Tharam S. Dillon, A Novel Adaptive Fuzzy Logic Controller (AFLC) to Reduce Retransmission and Service Roundtrip Time for Logical TCP Channels over the Internet, Proc. of the EU2004 Conference, August 2004, Japan, 942-951

A. Medina, I. Matta and J. Byers, On the Origin of Power Laws in Internet Topologies, ACM SIGCOMM, 30(2), $2000,18-28$
Wilfred W. K. Lin, Allan K. Y. Wong, and Tharam S Dillon, A Novel Fuzzy-PID Dynamic Buffer Tuning Model to Eliminate Overflow and Shorten the End-toEnd Roundtrip Time for TCP Channels, Lecture Notes in Computer Science, Springer Verlag LNCS Electronic Journal, http://www.springerlink.com/index/VF 155CH38XFL LB4H

S. Molnar, T.D. Dang and A. Vidacs, Heavy-Tailedness, Long-Range Dependence and Self-Similarity in Data Traffic, Proc. of the $7^{\text {th }}$ Int'l Conference on Telecommunication Systems, Modelling and Analysis, Nashville, USA, 18-21, 1999

H.O.Peitgen, H.Jurgens, D.Saupe, Chaos and Fractals: New Frontiers of Science $2^{\text {nd }}$ edition, Springer, 2004, pp. 686

J. Sarraille and P. DiFalco, FD3, http://life.bio.sunysb.edu/morph/fd3.html

M.S. Taqqu, Fractional Brownian Motion and LongRange Dependence, in Theory and Applications of Long-Range Dependence, P. Doukhan et al., Eds., Birkhuser 2003, 5-38

W. Willinger, V. Paxson, R.H. Hiedi and M.S. Taqqu, Long-Range Dependence and Data Network Traffic, in Theory and Applications of Long-Range Dependence, P. Doukhan et al., Eds., Birkhuser 2003, $373-408$

Allan K.Y. Wong and Joseph H.C. Wong, A Convergence Algorithm for Enhancing the Performance of Distributed Applications Running on Sizeable Networks, The International Journal of Computer Systems, Science \& Engineering, vol. 16, no. 4, July 2001, 229-236

Allan K.Y. Wong, Wilfred W.K. Lin, May T.W. Ip and Tharam S. Dillon, Genetic Algorithm and PD Control Together for Dynamic Anticipative Marginal Buffer Management: An Effective Approach to Enhance Dependability and Performance for Distributed Mobile Object-Based Real-time Computing over the Internet, Journal of Parallel and Distributed Computing (JPDC), vol.62, Sept. 2002, 1433-1453 

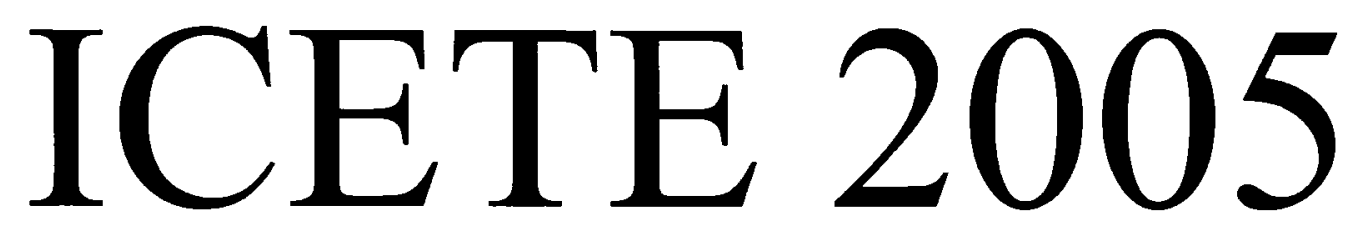

\author{
Proceedings of the \\ Second International Conference on \\ e-Business and Telecommunication Networks
}

Volume II

Reading, U.K.

October $3-7,2005$

Co-organized by

INSTICC - Institute for Systems and Technologies of Information, Control and Communication

Sponsored by Microsoft ${ }^{\circledR}$ and Escola Superior de Tecnologia de Setúbal

In Cooperation with The British Computer Society 
Gulden Uchyigit (Ed.)

\section{Web Personalisation, Recommender Systems and Intelligent User Interfaces}

Proceedings of the

1st International Workshop on

Web Personalisation, Recommender Systems

and Intelligent User Interfaces, WPRSIUI 2005

In conjunction with ICETE 2005

Reading, U.K., October 2005

INSTICC PRESS

Portugal 